\title{
Elovl6 is a poor prognostic predictor in breast cancer
}

\author{
YIN-HSUN FENG ${ }^{1,2}$, WEI-YU CHEN ${ }^{1}$, YU-HSUAN KUO ${ }^{1}$, CHAO-LING TUNG $^{1}$, \\ CHAO-JUNG TSAO ${ }^{3}$, AI-LI SHIAU ${ }^{4}$ and CHAO-LIANG WU ${ }^{5}$ \\ ${ }^{1}$ Division of Hematology and Oncology, Department of Internal Medicine, Chi-Mei Medical Center, Tainan 71004; \\ ${ }^{2}$ Department of Nursing, College of Medicine and Life Science, Chung Hwa University of Medical Technology, \\ Tainan 71703; ${ }^{3}$ Department of Hematology and Oncology, Chi-Mei Medical Center, Tainan 73657; \\ Departments of ${ }^{4}$ Microbiology and Immunology, and ${ }^{5}$ Biochemistry and Molecular Biology, \\ College of Medicine, National Cheng Kung University, Tainan 70101, Taiwan R.O.C.
}

Received March 28, 2015; Accepted April 29, 2016

DOI: $10.3892 / 01.2016 .4587$

\begin{abstract}
Elongation of long chain fatty acids family member 6 (Elov16) has been demonstrated to be involved in insulin resistance, obesity and lipogenesis. In addition, it has been reported that the protein is upregulated in human hepatocellular carcinoma and is implicated in nonalcoholic steatohepatitis-associated liver carcinogenesis. Excess body weight has been associated with an increased risk of postmenopausal breast cancer and poor prognosis. However, the connection between Elovl6 expression and outcome of breast cancer remains uncertain. Therefore, the present study used immunohistochemical analysis to investigate the expression of Elovl6 in breast cancer tissues from patients who had undergone curative mastectomy. Out of a total of 70 patients, $37.1 \%$ of patients exhibited positive Elovl6 expression in breast cancer tissue, whilst $62.9 \%$ were considered as negative. Positive Elov16 expression correlated with positive lymph node involvement and shorter recurrence-free survival. However, Elovl6 expression had no association with primary tumor size, lymph node metastasis, stage, grade, estrogen receptor, progesterone receptor, HER2 and age. Therefore, positive
\end{abstract}

Correspondence to: Dr Yin-Hsun Feng, Division of Hematology and Oncology, Department of Internal Medicine, Chi-Mei Medical Center, 901 Chung-Hwa Road, Yong Kang, Tainan 71004, Taiwan R.O.C.

E-mail: yinhsun.feng@gmail.com

Professor Chao-Liang Wu, Department of Biochemistry and Molecular Biology, College of Medicine, National Cheng Kung University, 1 Dashiue Road, Tainan 70101, Taiwan R.O.C.

E-mail: wumolbio@mail.ncku.edu.tw

Abbreviations: HCC, hepatocellular carcinoma; SREBP1, sterol regulatory element-binding protein 1; FAS, fatty acid synthase; NASH, non-alcoholic steatohepatitis; ACLY, ATP citrate lyase

Key words: elongation of long-chain fatty acids family member 6, breast cancer, prognosis, obesity, lipogenesis
Elovl6 expression is a poor prognostic factor in patients with breast cancer that have previously undergone surgery, and may function as a potential therapeutic approach in the future, particularly in the scope of obesity related disease.

\section{Introduction}

Overweight or obese patients have become an emerging health concern worldwide, and are associated with several diseases, including cardiovascular disease, type 2 diabetes mellitus and various forms of cancer (1). The prevalence of obesity has substantially increased over the last few decades, with the World Health Organization estimating that 500 million adults worldwide and $31 \%$ of females in the United States are categorically obese $(2,3)$. In Taiwan, the prevalence of obesity has increased to $13.2 \%$ of adult women, which poses a major task in the prevention of female cance (4). There is accumulating evidence that being overweight carries an established risk for renal cell cancer, colon cancer, endometrial cancer, esophageal adenocarcinoma and postmenopausal breast cancer. A major review by the International Agency for Research on Cancer analyzed data regarding weight, physical activity and cancer incidence in Europe (5). The review concluded that obesity contributed to the cause of $39 \%$ of endometrial cancer cases, $37 \%$ of esophageal cancer cases, $25 \%$ of kidney cancer cases, $11 \%$ of colon cancer cases and $9 \%$ of postmenopausal breast cancer cases (5). Data published over the last 25 years has indicated that obesity is responsible for $\sim 20 \%$ of cancer-associated mortalities in women and $\sim 14 \%$ in men (6). These rates are second only to smoking for the number of avoidable cases of cancer (6).

In women, breast cancer is the most frequently diagnosed form of cancer and the second highest cause of cancer-associated mortality in the United States, with a similar outcome reported for Asia-Pacific populations (7,8). Various factors associated with a greater risk of breast cancer have been identified. Among the modifiable risk factors, diet and obesity have been evaluated for use in strategies for breast cancer prevention. Previous studies have demonstrated that insulin resistance and diabetes mellitus contribute to cytotoxic agent resistance in cancer cells and poor response to chemotherapy in 
patients with hepatocellular carcinoma (HCC) $(9,10)$. Together with insulin resistance and diabetes mellitus, weight gain in adults is correlated with a greater risk of breast cancer and is a poor prognostic parameter, particularly in nonsmoking women (11-13). The mechanism underlying the association between increased incidences of breast cancer and obesity in women remains poorly understood, but a growing body of evidence suggests that insulin resistance, chronic inflammation, estrogen and adipokine interaction may be involved (14). An expression array analysis of breast tumor tissues from postmenopausal women demonstrated that progesterone receptor (PR) expression correlated with metabolic upregulation of glucolysis and lipogenesis (15). Furthermore, it has been identified that obesity is correlated with estrogen receptor (ER) and PR expression, thus supporting their underlying associations (16).

Processes related to cancer metabolism, such as glucolysis and lipogenesis, are part of a large body of research that may be able to define its exact role in cancer cells. However, the role of lipogenesis in tumor initiation remains unknown. In cancer stem cells of the breast, ectopic expression of sterol regulatory element-binding protein-1 (SREBP-1), which is a master regulator of lipogenic genes, promoted cell growth and mammosphere formation, and significantly enhanced lipogenesis in stem cells (17). Multifunctional fatty acid synthase (FAS) enzymes, which convert acetyl CoA into fatty acids, is overexpressed in a wide range of human tumors (18). Under hypoxic conditions, FAS is upregulated following activation of Akt and SREBP-1 (19). FAS expression in breast cancer confers poor prognosis and is associated with HER2 expression in aggressive breast cancer $(20,21)$, whilst FAS inhibition reverses the resistance of trastuzumab in HER2(+) breast cancer cells (22). As a long fatty acid elongase, Elovl6 contributes to de novo synthesis of fatty acids, and is understood to modulate insulin resistance (23). As well as FAS involved in de novo lipogenesis, the study of Elovl6 in carcinogenesis remains limited. In nonalcoholic steatohepatitis (NASH)-associated HCC, the expression of Elovl6 is upregulated, thus highlighting the contribution of lipogenesis in liver carcinogenesis (24). The aim of the present study is to nvestigate the behavior of Elovl6 in breast cancer, as it is important to understand the molecular mechanism of lipogenesis in mammary carcinogenesis, with ongoing efforts required to identify novel diagnostic and therapeutic targets.

\section{Materials and methods}

Patients and tumor samples. In 2006 and 2007, a total of 70 patients with histologically confirmed breast cancer were treated at Chi-Mei Medical Center (Tainan, Taiwan). All patients received standard therapy for curative purpose of breast cancer as indicated. Clinical information was obtained from medical records, and all samples were obtained by mastectomy. Specimens were fixed with $10 \%$ formalin and embedded in paraffin. The present study was approved by the institute review board of Chi-Mei Medical Center (institutional review board serial no. 10207-001).

Immunohistochemistry. Staining was carried out on formalin-fixed and paraffin-embedded tissue sections using immunoperoxidase methods. Following deparaffinization in xylene and rehydration in a graded alcohol series, the sections were heated in a microwave with citrate buffer for $13 \mathrm{~min}$ for heat-induced epitope retrieval (Thermo Fisher Scientific, Inc., Waltham, MA, USA). The following steps were performed using a Novolink ${ }^{\mathrm{TM}}$ Polymer Detection system (Leica Microsystems, Ltd., Milton Keynes, UK). Novolink Peroxidase Block was added for $5 \mathrm{~min}$ to neutralize the endogenous peroxidase activity, followed by the addition of Novolink Protein Block for 5 min. Next, the sections were incubated with the primary antibody against Elovl6 (dilution, 1:20; Atlas Antibodies, Stockholm, Sweden) overnight at $4^{\circ} \mathrm{C}$. The sections were washed and subsequently incubated with Novolink Post Primary Block for $10 \mathrm{~min}$, followed by Novolink Polymer for $10 \mathrm{~min}$. The color reaction was developed using NovoLink DAB Substrate Buffer, and the sections were counterstained with Mayer's hematoxylin (ScyTek Laboratories Inc., Logan, UT, USA). The intensity of the reactions were analyzed qualitatively. Microscopic fields with the highest degree of immunoreactivity were selected for analysis. The intensity score represented the mean staining intensity of the positive tumor cells, and was classified as follows: Negative, 0 ; weak, 1; moderate, 2; and strong, 3 , as described previously $(25,26)$. Intensity scores of 0 and 1 were considered to represent negative Elov16 expression, whilst scores of 2 and 3 were considered to represent positive Elovl6 expression.

Statistical analysis. All analyses were performed using SigmaStat version 3.1 (Systat Software, Inc., San Jose, CA, USA) and SPSS version 12.0 (SPSS, Inc., Chicago, IL, USA). The $\chi^{2}$ test was used to compare categorical variables, and Kaplan-Meier survival analysis was used to estimate survival curves. In addition, differences between two groups were analyzed using the Wilcoxon rank-sum test. All tests were two-tailed and $\mathrm{P}<0.05$ was considered to indicate a statistically significant difference.

\section{Results}

A total of 70 women with invasive breast cancer were included in the present study. All patients underwent mastectomy and indicated adjuvant treatment. The tumor samples were harvested from primary breast tumors. Among the 70 samples, $37.1 \%$ exhibited positive Elovl6 expression and $62.9 \%$ were considered as negative. Positive Elovl6 expression was defined as moderate to strong intensity from immunohistochemistry staining (Fig. 1). In high-power field microscopic analysis, the breast cancer cells exhibited positive expression of Elovl6 with cytoplasmic and nuclear distribution (Fig. 2). In order to analyze the contribution of Elovl6 to the development of breast cancer, the present study evaluated the association between Elovl6 expression in clinical breast tumor tissues and clinicopathological parameters, including primary tumor size, lymph node metastasis, stage, grade, ER, PR, HER 2 and age. The results demonstrated that positive Elov16 expression was correlated with positive lymph node involvement $(\mathrm{P}=0.018)$ (Table I). Positive lymph node involvement is known to function as an important predictor of breast cancer patient survival. In the present study, 8/70 patients experienced 


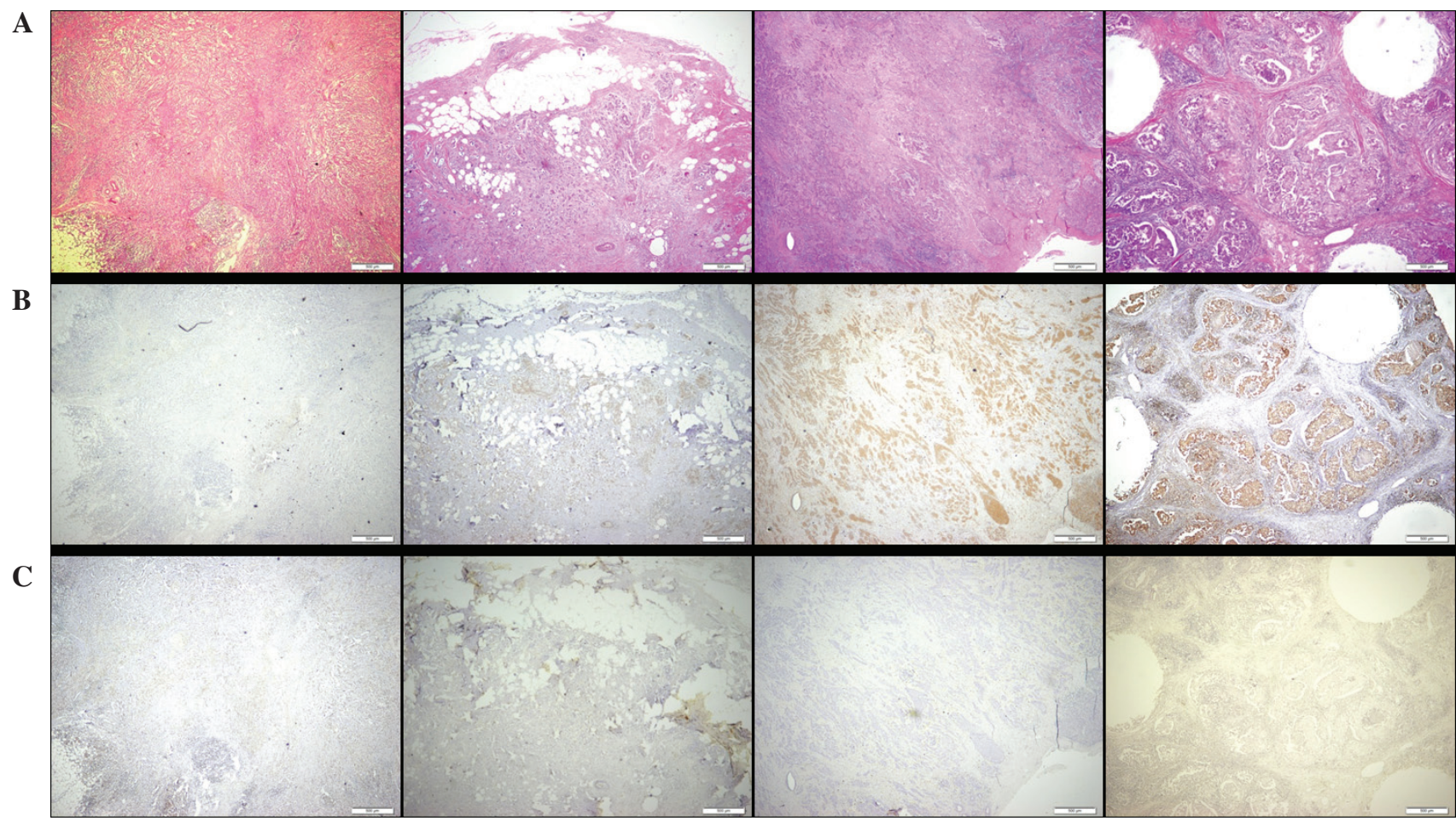

Figure 1. Representative examples of Elovl6 expression in human breast cancer. Left lane, intensity score 0; middle left lane, intensity score 1; middle right lane, intensity score 2; and right lane, intensity score 3. (A) Hematoxylin, (B) Elovl6 staining, and (C) negative control. Scale bars, 500 $\mu \mathrm{m}$. Elov16, elongation of long-chain fatty acids family member 6 .
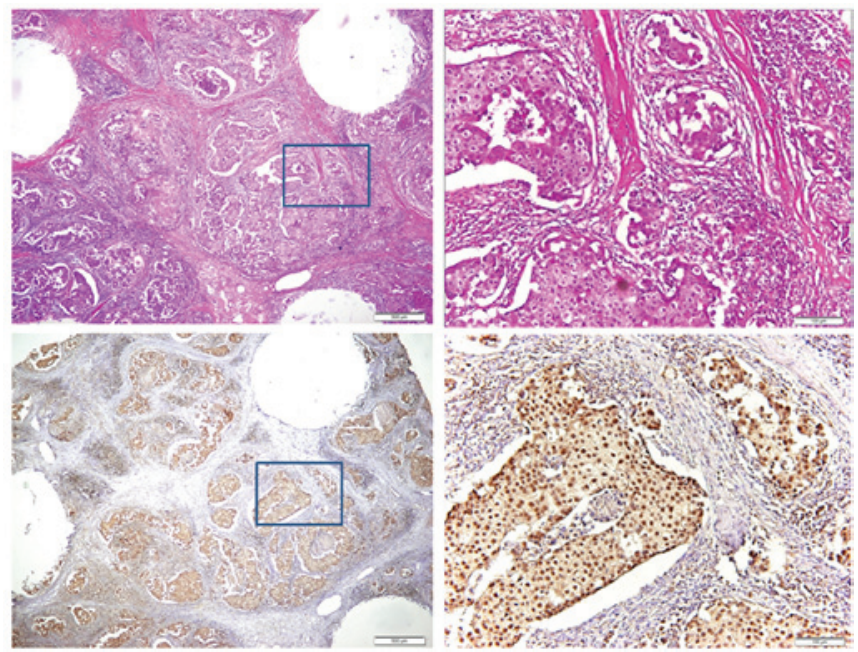

Figure 2. Histological detection of Elovl6 protein in breast cancer cells Lower panel, hematoxylin; upper panel, Elovl6 stain. Scale bars, $500 \mu \mathrm{m}$ (left) and $100 \mu \mathrm{m}$ (right). Elov16, elongation of long-chain fatty acids family member 6.

recurrence within 5 years of breast cancer resection. A total of 4/26 (15.4\%) patients with positive Elovl6 expression experienced recurrence compared with 4 out of 44 (9.1\%) patients with negative Elovl6 expression (Table II). The present study performed Kaplan-Meier survival analysis to estimate the recurrence-free survival time of patients with Elov16(+) breast cancer. The results demonstrated that patients with positive Elovl6 expression had a poorer recurrence-free survival time compared with patients with negative Elov16 expression $(\mathrm{P}<0.05)$ (Fig. 3).

\section{Discussion}

It has been reported that obesity increases the incidence, progression and mortality associated with breast cancer primarily in postmenopausal women $(27,28)$. A rodent model study demonstrated that obese animals deposited excess nutrients into tumors, and increased expression of PR was positively correlated with glycolytic and lipogenic enzymes from tumors (15). In a retrospective study in Asia, it was observed that obese women experience more advanced disease with higher axillary lymph node ratio and higher stage at diagnosis (16). The present study identified that Elovl6, a key enzyme involved in the lipogenesis pathway, functioned as a novel prognostic factor in breast cancer and was correlated with nodal metastasis. These findings support a previous hypothesis suggesting that the activation of fatty acid synthesis is required for tumor survival and carcinogenesis. Enzymes implicated in fatty acid synthesis may serve as a rational therapeutic target for cancer treatment (29). Due to the limited number of study samples, the present study is unable to clarify the prognostic role of Elovl6 in hormone positive breast cancer. Therefore, further studies with larger sample sizes are warranted.

Lipogenesis is considered as a potential target for the treatment of cancer and several enzymes involved in this process have been considered as therapeutic targets. The regulation of FAS in cancer was initially investigated in the 1980s in human breast cancer cells expressing functioning ERs and PRs (30). High expression of FAS has been associated with a greater risk of breast cancer-associated mortality, which has resulted in the investigation of FAS as a chemotherapy target (31). Orlistat, an inhibitor of FAS, was originally regarded as an antiobesity drug, but has now been identified to exert anticancer activity 
Table I. Association between Elovl6 expression and clinicopathological characteristics.

\begin{tabular}{|c|c|c|c|c|}
\hline Characteristic & $\mathrm{N}^{\mathrm{a}}$ & Elovl6 $(+)^{\mathrm{b}}$ & Elovl6 (-) $)^{\mathrm{c}}$ & P-value \\
\hline Tumor size & & & & 0.239 \\
\hline$<2 \mathrm{~cm}$ & 30 & 14 & 16 & \\
\hline$\geq 2 \mathrm{~cm}$ & 40 & 12 & 28 & \\
\hline Node involvement & & & & 0.018 \\
\hline Positive & 29 & 16 & 13 & \\
\hline Negative & 41 & 10 & 31 & \\
\hline Stage & & & & 0.298 \\
\hline I & 23 & 11 & 12 & \\
\hline II & 35 & 13 & 22 & \\
\hline III & 12 & 2 & 10 & \\
\hline Grade & & & & 0.295 \\
\hline $\mathrm{I}$ & 18 & 7 & 11 & \\
\hline II & 37 & 14 & 23 & \\
\hline III & 15 & 5 & 10 & \\
\hline ER & & & & 0.412 \\
\hline Positive & 40 & 17 & 23 & \\
\hline Negative & 30 & 9 & 21 & \\
\hline PR & & & & 0.186 \\
\hline Positive & 40 & 18 & 22 & \\
\hline Negative & 30 & 8 & 22 & \\
\hline HER2 & & & & 0.960 \\
\hline Positive & 28 & 10 & 18 & \\
\hline Negative & 42 & 16 & 26 & \\
\hline Diabetes & & & & 0.915 \\
\hline Positive & 17 & 6 & 11 & \\
\hline Negative & 53 & 20 & 33 & \\
\hline Overweight & & & & 0.062 \\
\hline Positive & 24 & 13 & 11 & \\
\hline Negative & 46 & 13 & 33 & \\
\hline
\end{tabular}

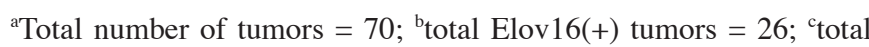
Elov16(-) tumors $=44$. Elov16, elongation of long-chain fatty acids family member 6; ER, estrogen receptor; PR, progesterone receptor; HER2, human epidermal growth factor receptor 2 .

in oral squamous cell carcinoma (32). Aside from extensive studies examining insulin resistance and lipogenesis, a limited amount of research has focused on the role of Elovl6 in cancer biology. In a study investigating pediatric primary central nervous system germ cell tumors, the expression of Elovl6 mRNA was observed to be abundant in germinoma (33). To analyze the oncogenic role of Elovl6, a phosphatase and tensin homolog-null mouse model demonstrated that Elovl6 expression was significantly higher in HCC tissue compared with adjacent NASH liver tissue (24). Among the enzymes involved in de novo lipogenesis, inhibition of ATP citrate lyase (ACLY) results in apoptosis and growth suppression in human cancer cells (34). In addition, ACLY depletion induces phosphorylation of AMP-activated protein kinase and coincides with Elov16 downregulation (35). Notably, it was reported that
Table II. Breast cancer recurrence rate at 5 years post-resection.

5-year disease

\begin{tabular}{llr} 
Characteristic & $\mathrm{N}$ & recurrence rate, \% (n) \\
\hline All tumors & 70 & $11.4(8)$ \\
Elov16(+) & 26 & $15.4(4)$ \\
Elovl6(-) & 44 & $9.1(4)$ \\
ER(+) tumors & 40 & $7.5(3)$ \\
Elov16(+) & 17 & $11.8(2)$ \\
Elov16(-) & 23 & $4.3(1)$ \\
PR(+) tumors & 40 & $10(4)$ \\
Elov16(+) & 18 & $11.1(2)$ \\
Elovl6(-) & 22 & $9.1(2)$
\end{tabular}

Elov16, elongation of long-chain fatty acids family member 6; ER, estrogen receptor; $\mathrm{PR}$, progesterone receptor.
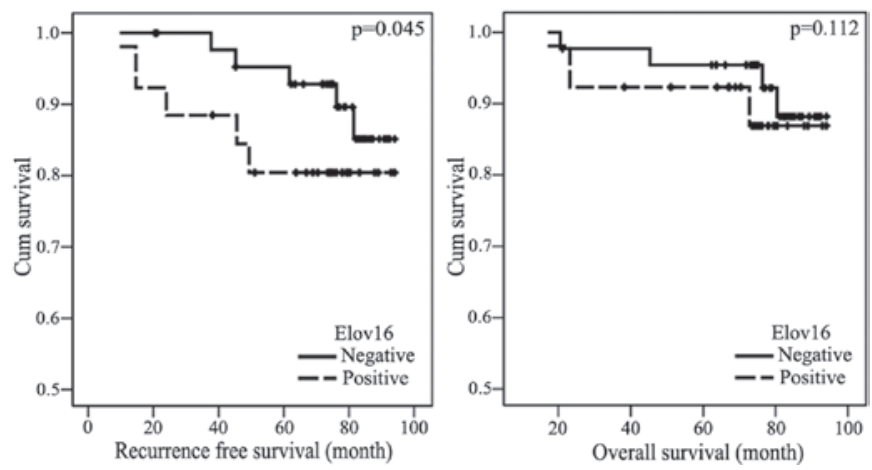

Figure 3. Survival in Elovl6(+) and Elovl6(-) breast cancer tissues as determined by Kaplan-Meier survival analysis. Elov16, elongation of long-chain fatty acids family member 6 .

diethylnitrosamine-induced carcinogenesis, which mimics inflammatory events in early hepatocarcinogenesis, increased C18/C16 ratio and hepatic lipids. To clarify the emerging role of Elovl6 in cancer biology, a recent study analyzed human liver samples from patients with NASH or NASH-related HCC (36). The results demonstrated that the NASH and NASH-related HCC tissues exhibited an elevated expression of Elovl6 (36). When combined, these results suggest that Elovl6 may be regarded as an oncogenic protein in the process of lipogenesis.

Elovl6 deficiency reduces SREBP-1 and peroxisome proliferator-activated receptor $\alpha$, in addition to altering hepatic fatty acid composition. Elovl6 knockout mice are unique as their insulin resistance increases without amelioration of hepatosteatosis and obesity (37). This emphasizes the importance of tissue fatty acid composition in insulin sensitivity, which may be controlled by Elovl6 activity (37). A novel, selective and potent active inhibitor for mammalian Elovl6 has been identified. In a previous study, chronic treatment with an oral inhibitor of Elovl6 in animals with diet-induced obesity resulted in a reduction in hepatic fatty composition (38). However, the same inhibitor failed to improve insulin sensitivity via Elovl6 inhibition in several other animal models (38). 
Based on the implication of Elovl6 expression in breast cancer, further studies are required to evaluate the therapeutic potential of Elovl6 inhibition in breast cancer treatment.

In conclusion, the present study demonstrated that Elovl6, a microsomal enzyme that regulates fatty acid metabolism and insulin sensitivity, was associated with a poor outcome in patients with operable breast cancer. In addition, the overexpression of Elovl6 was associated with malignant involvement of regional lymph nodes and shorter recurrence free survival. These results suggest that Elovl6 may function as a prognostic predictor in human breast cancer and may hold promise as a potential strategy for cancer chemoprevention and treatment.

\section{Acknowledgements}

The project was funded in part by Chi-Mei Medical Center, Tainan, Taiwan (grant nos. CMNCKU10004 and CMHFR10204).

\section{References}

1. Andersen DK: Diabetes and cancer: Placing the association in perspective. Curr Opin Endocrinol Diabetes Obes 20: 81-86, 2013.

2. Sundaram S, Johnson AR and Makowski L: Obesity, metabolism and the microenvironment: Links to cancer. J Carcinog 12: 19, 2013.

3. Ogden CL, Carroll MD, Curtin LR, McDowell MA, Tabak CJ and Flegal KM: Prevalence of overweight and obesity in the United States, 1999-2004. JAMA 295: 1549-1555, 2006.

4. Chu NF: Prevalence of obesity in Taiwan. Obes Rev 6: 271-274, 2005.

5. Bergström A, Pisani P, Tenet V, Wolk A and Adami HO: Overweight as an avoidable cause of cancer in Europe. Int J Cancer 91: 421-430, 2001.

6. Calle EE, Rodriguez C, Walker-Thurmond $\mathrm{K}$ and Thun MJ: Overweight, obesity and mortality from cancer in a prospectively studied cohort of U.S. adults. N Engl J Med 348: 1625-1638, 2003

7. Jemal A, Siegel R, Ward E, Hao Y, Xu J, Murray T and Thun MJ: Cancer statistics, 2008. CA Cancer J Clin 58: 71-96, 2008.

8. Renehan AG, Tyson M, Egger M, Heller RF and Zwahlen M: Body-mass index and incidence of cancer: A systematic review and meta-analysis of prospective observational studies. Lancet 371: 569-578, 2008.

9. Feng YH, Velazquez-Torres G, Gully C, Chen J, Lee MH and Yeung SC: The impact of type 2 diabetes and antidiabetic drugs on cancer cell growth. J Cell Mol Med 15: 825-836, 2011.

10. Feng YH, Lin CY, Huang WT, Wu CL, Fang JL, et al: Diabetes mellitus impairs the response to intra-arterial chemotherapy in hepatocellular carcinoma. Med Oncol 28: 1080-1088, 2011.

11. Coates RJ, Clark WS, Eley JW, Greenberg RS, Huguley CM Jr and Brown RL: Race, nutritional status, and survival from breast cancer. J Natl Cancer Inst 82: 1684-1692, 1990.

12. Caan BJ, Kwan ML, Hartzell G, Castillo A, Slattery ML, Sternfeld B and Weltzien E: Pre-diagnosis body mass index, post-diagnosis weight change, and prognosis among women with early stage breast cancer. Cancer Causes Control 19: 1319-1328, 2008.

13. Kroenke CH, Chen WY, Rosner B and Holmes MD: Weight, weight gain, and survival after breast cancer diagnosis. J Clin Oncol 23: 1370-1378, 2005.

14. Okwan-Duodu D, Umpierrez GE, Brawley OW and Diaz R: Obesity-driven inflammation and cancer risk: Role of myeloid derived suppressor cells and alternately activated macrophages. Am J Cancer Res 3: 21-33, 2013.

15. Giles ED, Wellberg EA, Astling DP, Anderson SM, Thor AD, Jindal S, Tan AC, Schedin PS and Maclean PS: Obesity and overfeeding affecting both tumor and systemic metabolism activates the progesterone receptor to contribute to postmenopausal breast cancer. Cancer Res 72: 6490-6501, 2012.

16. Kaviani A, Neishaboury M, Mohammadzadeh N, Ansari-Damavandi, et al: Effects of obesity on presentation of breast cancer, lymph node metastasis and patient survival: A retrospective review. Asian Pac J Cancer Prev 14: 2225-2229, 2013.
17. Pandey PR, Xing F, Sharma S, Watabe M, Pai SK, Iiizumi-Gairani M, Fukuda K, Hirota S, Mo YY and Watabe K: Elevated lipogenesis in epithelial stem-like cell confers survival advantage in ductal carcinoma in situ of breast cancer. Oncogene 32: 5111-5122, 2013.

18. Kuhajda FP, Jenner K, Wood FD, Hennigar RA, Jacobs LB, Dick JD and Pasternack GR: Fatty acid synthesis: A potential selective target for antineoplastic therapy. Proc Natl Acad Sci USA 91: 6379-6383, 1994

19. Furuta E, Pai SK, Zhan R, Bandyopadhyay S, Watabe M, Mo YY, Hirota S, Hosobe S, Tsukada T, Miura K, et al: Fatty acid synthase gene is up-regulated by hypoxia via activation of Akt and sterol regulatory element binding protein-1. Cancer Res 68: 1003-1011, 2008.

20. Alo' PL, Visca P, Marci A, Mangoni A, Botti C and Di Tondo U: Expression of fatty acid synthase (FAS) as a predictor of recurrence in stage I breast carcinoma patients. Cancer 77: 474-482, 1996.

21. Zhang D, Tai LK, Wong LL, Chiu LL, Sethi SK and Koay ES: Proteomic study reveals that proteins involved in metabolic and detoxification pathways are highly expressed in HER-2/neu-positive breast cancer. Mol Cell Proteomics 4: 1686-1696, 2005.

22. Vazquez-Martin A, Colomer R, Brunet J and Menendez JA: Pharmacological blockade of fatty acid synthase (FASN) reverses acquired autoresistance to trastuzumab (Herceptin by transcriptionally inhibiting 'HER2 super-expression' occurring in high-dose trastuzumab-conditioned SKBR3/Tzb100 breast cancer cells. Int J Oncol 31: 769-776, 2007.

23. Matsuzaka T, Shimano H, Yahagi N, Kato T, Atsumi A, Yamamoto T, Inoue N, Ishikawa M, Okada S, Ishigaki N, et al: Crucial role of a long-chain fatty acid elongase, Elovl6, in obesity-induced insulin resistance. Nat Med 13: 1193-1202, 2007.

24. Muir K, Hazim A, He Y, Peyressatre M, Kim DY, Song X and Beretta L: Proteomic and lipidomic signatures of lipid metabolism in NASH-associated hepatocellular carcinoma. Cancer Res 73: 4722-4731, 2013.

25. Cheng L, Nagabhushan M, Pretlow TP, Amini SB and Pretlow TG: Expression of E-cadherin in primary and metastatic prostate cancer. Am J Pathol 148: 1375-1380, 1996.

26. Cheng L, Pan CX, Zhang JT, Zhang S, Kinch MS, Li L, Baldridge LA, Wade C, Hu Z, Koch MO, et al: Loss of 14-3-3sigma in prostate cancer and its precursors. Clin Cancer Res 10: 3064-3068, 2004.

27. Huang Z, Willett WC, Colditz GA, Hunter DJ, Manson JE, Rosner B, Speizer FE and Hankinson SE: Waist circumference, waist:hip ratio, and risk of breast cancer in the Nurses' Health Study. Am J Epidemiol 150: 1316-1324, 1999.

28. Reeves GK, Pirie K, Beral V, Green J, Spencer E and Bull D; Million Women Study Collaboration: Cancer incidence and mortality in relation to body mass index in the Million Women Study: Cohort study. BMJ 335: 1134, 2007.

29. Zaidi N, Lupien L, Kuemmerle NB, Kinlaw WB, Swinnen JV and Smans K: Lipogenesis and lipolysis: The pathways exploited by the cancer cells to acquire fatty acids. Prog Lipid Res 52: 585-589, 2013.

30. Chalbos D, Joyeux C, Galtier F, Escot C, Chambon M, Maudelonde $\mathrm{T}$ and Rochefort $\mathrm{H}$ : Regulation of fatty acid synthetase by progesterone in normal and tumoral human mammary glands. Rev Esp Fisiol 46: 43-46, 1990.

31. Wang Y, Kuhajda FP, Li JN, Pizer ES, Han WF, Sokoll LJ and Chan DW: Fatty acid synthase (FAS) expression in human breast cancer cell culture supernatants and in breast cancer patients. Cancer Lett 167: 99-104, 2001.

32. Agostini M, Almeida LY, Bastos DC, Ortega RM, Moreira FS, Seguin F, Zecchin KG, Raposo HF, Oliveira HC, Amoêdo ND, et al: The fatty acid synthase inhibitor orlistat reduces the growth and metastasis of orthotopic tongue oral squamous cell carcinomas. Mol Cancer Ther 13: 585-595, 2014.

33. Wong JM, Chi SN, Marcus KJ, Levine BS, Ullrich NJ, MacDonald S, Lechpammer $M$ and Goumnerova LC: Germinoma with malignant transformation to nongerminomatous germ cell tumor. J Neurosurg Pediatr 6: 295-298, 2010.

34. Migita T, Okabe S, Ikeda K, Igarashi S, Sugawara S, et al: Inhibition of ATP citrate lyase induces an anticancer effect via reactive oxygen species: AMPK as a predictive biomarker for therapeutic impact. Am J Pathol 182: 1800-1810, 2013. 
35. Migita T, Okabe S, Ikeda K, Igarashi S, Sugawara S, Tomida A, Soga T, Taguchi R and Seimiya H: Inhibition of ATP citrate lyase induces triglyceride accumulation with altered fatty acid composition in cancer cells. Int J Cancer 135: 37-47, 2014.

36. Kessler SM, Simon Y, Gemperlein K, Gianmoena K, Cadenas C, Zimmer V, Pokorny J, Barghash A, Helms V, van Rooijen N, et al: Fatty acid elongation in non-alcoholic steatohepatitis and hepatocellular carcinoma. Int J Mol Sci 15: 5762-5773, 2014.
37. Matsuzaka $\mathrm{T}$ and Shimano H: Elovl6: A new player in fatty acid metabolism and insulin sensitivity. J Mol Med (Berl) 87: 379-384, 2009

38. Shimamura K, Nagumo A, Miyamoto Y, Kitazawa H, Kanesaka M, Yoshimoto R, Aragane K, Morita N, Ohe T, Takahashi T, et al: Discovery and characterization of a novel potent, selective and orally active inhibitor for mammalian ELOVL6. Eur J Pharmacol 630: 34-41, 2010. 